\title{
Affective Recognition for Multimedia Environments: A Review
}

\author{
Mohammadhossein Moghimi ${ }^{1}$, Robert Stone ${ }^{2}$ and Pia Rotshtein ${ }^{3}$ \\ ${ }^{1,2}$ School of Electronic, Electrical and Systems Engineering, University of Birmingham \\ ${ }^{3}$ School of Psychology, University of Birmingham \\ 1m.moghimi@pgr.bham.ac.uk, ${ }^{2}$ r.j.stone@bham.ac.uk, ${ }^{3}$ p.rotshtein@bham.ac.uk
}

\begin{abstract}
Detecting emotional responses in multimedia environments is an academically and technologically challenging research issue. In the domain of Affective Computing, from non-interactive and static stimuli (e.g. affective image) to highly interactive and dynamic environments (affective virtual realities), researchers have employed a wide range of affective stimuli to measure and interpret human psychological and physiological emotional behaviours. Various psychophysiological parameters (e.g. Electroencephalography, Galvanic Skin Response, Heart Rate, etc.) have been employed and investigated, in order to detect and quantify human affective states. In this paper, we present a detailed literature review of over 33 affective computing studies, undertaken since 1993. All aspects of these studies (stimuli type, pre-processing, windowing, features, classification technique, etc.) have been reported in detail. We believe that this paper not only summarises the breadth of research over the past 20 years, but also serves to clarify various significant aspects and details of this increasingly valuable and relevant research area.
\end{abstract}

Keywords - Affective Computing, Emotion Recognition, Multimedia Systems, Brain Computer Interaction, Model of Emotions, Affective Space

\section{Introduction}

motions play an important role in the day-

$\mathrm{E}$ to-day life of people. These psychophysiological processes can be elicited by conscious or/and unconscious perception of situations, events or objects, and may be influenced by an individual's previous experiential history, including exposure to traumatic incidents. Emotions, or so-called affective experiences, can be expressed either verbally or through overt and subtle behaviours. Moreover, emotions impact physiological, neurophysiological and cognitive responses as well, as part of a complex process that enables the individual to react and perform appropriately to personal events. Expressing and comprehending emotional states of people are not only important at a personal level, but can also affect the behaviour of others in different circumstances [1]. Therefore, prediction of type and intensity of emotional experiences plays an extremely important role in human social life.

On the other hand, the growing use of innovative Human-Computer Interactive (HCI) devices (e.g. computers, Smartphones, wearable smart technologies, etc.) has raised a new demand whereby users of such technologies seem to be increasingly able to exchange psychological and cognitive states with mobile and online monitoring and archiving systems. This (relatively) new and challenging scientific frontier is often referred to as Brain-Computer
Interaction (BCI). BCI systems attempt to improve human-computer interaction by interfacing directly with the human nervous system (both central and autonomic), thereby, it is claimed, removing the artificial barriers to intuitive interaction typically experienced when using conventional input-display techniques. Researchers in the field of Affective computing have employed BCI techniques to identify and categorise emotional experiences, with the ultimate aim of enabling advanced HCI systems to adapt their functions according to human affective states. This new channel for human interaction is demonstrating great potential for improving the future performance of $\mathrm{HCI}$ systems, employed for various applications [2].

Affective computing has received a considerable amount of attention in the past two decades [2], [3], [4], [5], [6]. These studies have tried to evoke, identify and classify a number of emotional experiences, using various static (image-based) [5], dynamic (video and music) [3] and interactive (virtual reality (VR) and gamesbased scenario) [2] stimuli. Moreover, various physiological recordings (including Electroencephalography (EEG) [3] and Galvanic Skin Response (GSR) [2]) have been exploited by different researchers, in order to support the affective recognition process.

In this paper, as part of a larger research project, a general summary of the affective computing field and a detailed literature review of over 33 affective recognition studies, 
published since 1993, have been presented. In Section 2 of the present paper, the general phases of conceptualisation, design and evaluation of an affective recognition system have been discussed. In Section 3, all aspects of the reviewed affective computing studies (e.g. stimuli type, physiological signal pre-processing, filtering, windowing, feature extraction, etc.) have been organised and reported, in detail. Furthermore, in Section 4, various models of emotions (categorical vs. dimensional) have been reviewed, as presented in the psychology literature, and employed in affective recognition studies. Also, different types of emotion assessment processes, which have been employed by the studies to subjectively identify the human emotions, have been identified and reported in Section 4.

\section{Affective Recognition}

To measure and recognise the emotional content of affective stimuli, two distinct approaches have been employed in the literature:

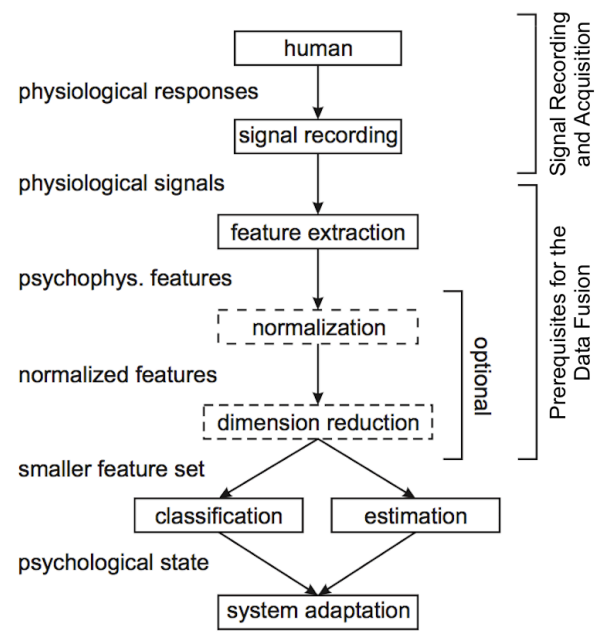

Figure 1 - The block diagram of general process of psychophysiological signal computing [7]

\section{Content-Centred}

Affective

Recognition: In this approach, the emotional effects of a number of particular stimuli (e.g. viewing images, performing specific tasks or games, recalling emotional memories, etc.) are subjectively assessed [7], [8], [9], [10], [11]. This recognition is performed through subjective assessments (e.g. Self-Assessment Manikin, SAM [12]) of a number of participants who have experienced the affective stimulations. As an illustration, a number of participants are instructed to observe a selection of images, which have been designed to evoke specific emotional experiences. Participants are required to report their emotions immediately after the exposure. As a result, the content-centred affective recognition process evaluates the emotional effect, power and efficiency of a number of particular stimuli, within a specific affective model (refer to Section 4.1).

\section{User-Centred Affective Recognition:}

In this approach, the affective psychophysiological behaviours of a number of participants (rather than their personal subjective assessments) are evaluated and classified, according to a number of emotional stimulations. This approach could be highly related to the content-centred affective recognition technique, as the performance of the user-centred affective recogniser is mainly evaluated through subjective assessments (through hypothesis, self or expert assessments - refer to Section 4.2). However, the technical and technological challenges of this approach, compare to the content-centred affective recognition, remain highly independent and different. Figure 1 shows the process of affective assessment and classification of psychophysiological signals in a workflow diagram [13]. As can be seen, a number of physiological signals are recorded from a number of participants, while exposed to a number of affective stimulations (refer to Section 3.1). Some pre-processing procedures may also be applied to the raw recorded signals, in order to attenuate the effects of noise and unwanted artefacts (refer to Section 3.2). Then, the data fusion process is performed through three steps. The feature extraction stage processes the incoming raw psychophysiological signals and extracts some physiologically relevant features to store them in a feature matrix, consisting of multiple valuable features (refer to Section 3.3). The feature matrix variability and dimensionality are attenuated through normalisation and feature selection or dimension reduction techniques. However, these steps could be skipped with respect to the variability and complexity of the feature matrix (refer to Section 3.4 and 3.5). Finally, the feature matrix is employed within estimation or classification processes to recognise the participants' emotional experiences (refer to Section 3.6). As stated in the final step, the participants' affective information can be employed within an 
advanced HCI system to adapt its environment and/or functionality accordingly.

The focus of this paper is to present and compare the employed techniques and conclusions of studies conducted previously, to perform User-Centred Affective Recognition (refer to Section 3); however, subjective affective assessment techniques, which have been employed to conduct performance evaluation, have also been discussed in detail (refer to Section 4).

\section{Related Research}

In this section, a deep review of 33 affective computing studies, conducted since 1993, has been presented. To review these studies appropriately, the evaluations have been divided into six sub-sections. Table 3 presents a summary of these sub-sections.

\subsection{Stimuli and Physiological Measurements}

To analyse the emotional response of humans and their psychophysiological responses, a psychophysiological affective database, recorded from a number of users exposed to a number of controlled and known affective stimuli, is required. To construct such a database, a number of controlled emotional scenarios (affective stimuli), evoking some specific affective states, on the part of the users ${ }^{1}$, need to be presented to participants in an experiment, whilst taking part in a physiological measurement paradigm. These recordings, tagged by the corresponding affective states, are analysed for the design, training and validation of the affective recognition system. Therefore, two distinct steps in the psychophysiological affective database construction can be considered: (I) evoking controlled emotional experiences and (II) the measurement of physiological parameters. It is important to ensure the implementation of strict experimental designs in such a paradigm, in order to avoid the development of an inappropriate psychophysiological affective database, which would invalidate the recognition system's training process. As an illustration, if the users' emotional experiences were poorly controlled (e.g. it was not possible to state with confidence that anger had been experienced by the users during the corresponding session), then the classification techniques would be unable to train

\footnotetext{
${ }^{1}$ Such as images that evoke fear and disgust on the part of users - image number 3000 to 3266 in IAPS [7]
}

the affective recognition system properly and the accuracy of the system would be compromised. To prevent such incidents, the authors argue that the emotional stimuli must be subjectively evaluated and categorised prior to the undertaking of physiological measurements, in order to validate their effectiveness in evoking the required emotional experiences on the part of all users. So far various affective stimuli types have been employed in order to perform human emotional experience assessment and classification (summarised in Table 1). These are:

1. Videos and Music Videos: The majority of the studies have concentrated on affect analysis and recognition, evoked during watching either videos (short clips from various movies scenes) [3], [14], [15], [16], [17], [18] (Employing MAHNOB [9] Affective Video Database), [19], [20], [21], or music videos (a short video integrating a combination of a particular song and imagery) [22], [23], [10] (Employing DEAP [10] Affective Music Video Database). The average videos and music videos duration was reported as 142.76 seconds $( \pm 144.7$, minimum 35 and maximum 591 seconds).

2. Images: A considerable number of studies used images as their affective stimuli, employed extracts from the International Affective Picture System database (IAPS [7]) [5], [6], [24], [25], [26], [27]; whilst a smaller number were found to employ other image datasets, such as affective face gestures [28]. The average image presentation duration was reported as 14.5 seconds $( \pm 22.88$, minimum 1 and maximum 60 seconds).

3. Sound: Studies, which used sound as their affective stimuli, employed either music [29], [30], or extracts from the International Affective Digital Sound database (IADS [8]) [31]. The average sound duration was reported as 210 seconds $( \pm 112.24$, minimum 60 and maximum 330 seconds).

4. Virtual Reality Scenarios and Games: A number of studies employed games and virtual reality scenes as their stimuli [2], [4], [32], [33], [34], [35]. The average games and VR duration was reported as 226 seconds $( \pm 106.44$, minimum 120 and maximum 350 seconds).

5. Real Life Scenario: There are some studies which employed real life scenarios (e.g. racing car driving, emotional situation 
recalling, etc.) to elicit and measure various emotional experiences [36], [37], [38], [39], [40]. The average stimulation duration was reported as 170 seconds $( \pm 75.5$, minimum 90 and maximum 240 seconds).

Different forms of physiological measurements have been implemented in the emotional experience assessment and classification research area. The majority of studies, reviewed, measured central nervous system signals, such as EEG [2], [3], [4], [5], $[10],[14],[15],[16],[18],[20],[21],[23],[24]$, [26], [28], [29], [33], [34], [40], and autonomic nervous system signals, such as heart rate (either through Electrocardiography (ECG) or Photoplethysmography (PPG) signals) [2], [6], [10], [17], [19], [21], [22], [23], [25], [29], [30],
[31], [35], [36], [37], [38], [39] and GSR [2], [5], $[6],[10],[17],[19],[21],[23],[25],[30],[32]$, [34], [35], [36], [37], [38], [39] to detect affective states. There are other autonomic nervous system signals, which have been employed by a minority of studies (when compared to EEG, heart rate and GSR), such as respiratory (breathing) rate [2], $[\mathbf{1 0}],[\mathbf{2 3}],[\mathbf{2 5}],[\mathbf{3 0}],[\mathbf{3 4}],[37],[\mathbf{3 8}]$, skin temperature [10], [17], [23], [25], [34], [35], [36], Electromyography (EMG) [6], [10], [23], [29], [30], [35], [37], [38], [39], blood pressure [17], [23], [34], pupil diameter [3], [36], eye movements [27] and Magnetoencephalography (MEG) [22]. Table 1 presents a summary of the affective stimuli and the physiological measurements employed by the researchers to perform affective recognition and classification.

Table 1 - Stimuli Type and Physiological Measurements Employed by Studies

\begin{tabular}{|c|c|}
\hline Stimuli Type & Used in Studies \\
\hline Video / Music Video & {$[3],[10],[14],[15],[16],[17],[18],[19],[20],[21],[22],[23]$} \\
\hline Image & {$[5],[6],[24],[25],[26],[27],[28]$} \\
\hline Virtual Reality and Games & {$[2],[4],[32],[33],[34],[35]$} \\
\hline Sound & {$[29],[30],[31]$} \\
\hline Real Life Scenarios & {$[36],[37],[38],[39],[40]$} \\
\hline Physiological Measurement & Used in Studies \\
\hline Electroencephalography (EEG) & $\begin{array}{c}{[2],[3],[4],[5],[10],[14],[15],[16],[18],[20],[21],[23],[24],[26],} \\
{[28],[29],[33],[34],[40]}\end{array}$ \\
\hline Heart Rate & $\begin{array}{c}{[2],[6],[10],[17],[19],[21],[22],[23],[25],[29],[30],[31],[35],} \\
{[36],[37],[38],[39]}\end{array}$ \\
\hline Galvanic Skin Response (GSR) & $\begin{array}{c}{[2],[5],[6],[10],[17],[19],[21],[23],[25],[30],[32],[34],[35],} \\
{[36],[37],[38],[39]}\end{array}$ \\
\hline Respiratory (Breathing) Rate & {$[2],[10],[23],[25],[30],[34],[37],[38]$} \\
\hline Skin Temperature & {$[10],[17],[23],[25],[34],[35],[36]$} \\
\hline Electromyography (EMG) & {$[6],[10],[23],[29],[30],[35],[37],[38],[39]$} \\
\hline Blood Pressure & {$[17],[23],[34]$} \\
\hline Pupil Diameter & {$[3],[36]$} \\
\hline Eye Movements & [27] \\
\hline Magnetoencephalography (MEG) & {$[22]$} \\
\hline
\end{tabular}

\subsection{Pre-processing (Artefact Removal and Filtering)}

The signals recorded from the central nervous system are highly susceptible to noise and unwanted artefacts [41]. In addition, all physiological signals recorded from the autonomic nervous system can be accompanied by unwanted noise. As an illustration, heart rate, measured using PPG, can carry unwanted noise due to harsh or rapid movements on the part of the subject's finger. Almost all studies reviewed in this paper did not apply any form of filtering on autonomic nervous system signals ${ }^{2}$. However, to eliminate noise in EEG signals, two key techniques were found to have been employed by those studies reviewed:

\footnotetext{
${ }^{2}$ Only [19] applied a band-pass filter $0.5 \mathrm{~Hz}$ to $35 \mathrm{~Hz}$ on heart rate signal, and a low-pass filter $10 \mathrm{~Hz}$ on GSR signal.
} 
1. Artefact Removal: Muscle, eye and head movements and blinking are the main sources of noise in EEG signals [41]. To minimise this type of noise, all reviewed studies instructed the participants to minimise movements as far as was possible, and to avoid excessive blinking. Half of the studies employed other artefact removal techniques, in addition, to minimise the effects of these types of noises even further. For example, $[2],[4], \quad[\mathbf{2 0}], \quad[\mathbf{2 3}], \quad[33]$ employed Electrooculography (EOG) signals to attenuate eye and muscle movement. [34], [40] employed visual checking techniques, to discard portions of the EEG signal with high muscular and eye movement noises. [5] and [10] adopted other artefact removal techniques to attenuate eye and muscle movements (e.g. Least Mean Square (LMS) and Blind Source Separation (BSS) algorithms).

2. Filtering: Applying low, high and band-pass filters are a common technique in reducing unwanted oscillations in a signal. To minimise noises in the EEG signals, all studies applied a band-pass filter to the raw signals, with various cut-off frequencies. The majority of studies set either $4 \mathrm{~Hz}$ or $5 \mathrm{~Hz}$ as the lower-band cut-off frequency [3], [4], [10], [14], [18], [23], [29], [34], whilst others employed a variety of even lower frequencies $(0.5 \mathrm{~Hz}, 1 \mathrm{~Hz}$, etc.) [2], [5], [15], [16], [20], [24], [33]. For the upper-band cut-off frequency, the majority of studies adopted $40 \mathrm{~Hz}$ or $45 \mathrm{~Hz}[\mathbf{3}],[\mathbf{4}],[\mathbf{5}],[\mathbf{1 0}],[\mathbf{1 4}],[\mathbf{1 5}]$, [18], [23], [29], [34], whilst others employed other frequencies $(30 \mathrm{~Hz}, 70 \mathrm{~Hz}$, etc.) [2], [16], $[\mathbf{2 0}],[24],[33],[40]$. This was due to the fact that eye-blinking artefacts are mainly observed in frequencies lower that $4 \mathrm{~Hz}$, as people rarely blink more than 4 times a second. Thus selecting $4 \mathrm{~Hz}$ as the lower-band cut-off frequency attenuates most of the blinking effects, present in the raw EEG signals. Moreover, the brain's high frequency rhythms (Gamma range) can be observed between $30 \mathrm{~Hz}$ and $45 \mathrm{~Hz}$ [42]. Therefore selecting $45 \mathrm{~Hz}$ as the upper-band cut-off frequency attenuates most of the higher unwanted frequencies.

\subsection{Windowing and Physiological Feature Extraction}

To perform affective recognition, a number of psychophysiological features need be extracted from the autonomic and/or central nervous system, while the participant is exposed to a number of affective stimuli (Section 3.1). These features need to be related to the affective states, as they will ultimately be employed within the affective recognition system to predict the emotional response of the users, when experiencing a specific affective situation. However, to train a supervised learner [43], to recognise the users' emotional experiences, the affective states of a number of participants, exposed to a number of affective stimuli, need to be properly pre-evaluated (refer to Section 4.2). This evaluation can then be used as the reference responses within the affect recogniser training process.

To train the emotion recognition agent (considering supervised learning algorithms [43]), a number of physiological features (all related to affective states) need to be extracted from the raw physiological signals. These features can be:

1. The statistical analysis (e.g. mean, standard deviation, etc.) of the raw signals (e.g. average GSR value, mean of the heart rate peaks, etc. [2], [38], [39]).

2. The frequency analysis of physiological signals to extract specific rhythms (e.g. alpha, beta, gamma rhythm power within the EEG signal [3], [30]).

3. The detection of specific patterns, such as Event Related Potentials (ERP - such as the P300, N100, and others [5], [20]).

4. Other exclusive measurements (e.g. $\left.\mathrm{EEG}_{\mathrm{w}}{ }^{9}[\mathbf{3 4}]\right)$.

Table 2 presents a list of affective features, employed by the reviewed studies, which have been reported to be related to the emotional states. Only the features extracted from the most commonly used physiological signals are covered here (EEG, GSR and Heart Rate - As reported in Section 3.1).

To extract the affective features (employing statistical, spectral, pattern or/and other measurements), a portion (called a Window) of the corresponding raw physiological signal is extracted and analysed. Any affective feature, extracted from this portion of the physiological signal, has to be able to be confidently tagged by a specific emotional experience. The emotionally labelled affective features, extracted from this period, are employed as a single observation, within the affective database, for the emotion 
recognition training process. Three main algorithms have been employed in the literature, to extract the appropriate periods of the physiological signals, which can be confidently tagged by a specific affective state:

\section{Window with Entire Stimuli Length:}

$40 \%$ of the reviewed studies extracted the features from the entire duration of the stimuli, regardless of the length. As an illustration, if participants were exposed to affective images for 5 seconds, the affective features were extracted from the 5-second affective period. Similarly, if participants were exposed to affective music videos for 60 seconds, the affective features were extracted from the entire 60 -second affective period [4], [15], [16], [17], [18], [24], [25], [28], [29], [33], [35], [39], [40].

2. Window Shorter than Stimuli Length: $36 \%$ of the reviewed studies extracted the features from multiple windows within each stimulus (i.e. shorter than the overall stimuli duration). The majority of these studies employed non-overlapped windowing techniques [2], [19], [20], [22], [30], [31], [32], [34], [37], and minority have used windows with more than $50 \%$ overlap [3], [14], [27].

3. Window Longer than Stimuli Length: only $15 \%$ of the studies included the post or/and pre-stimuli periods, and extracted the features from windows longer than the stimuli length [5], [6], [10], [23].

\subsection{Normalisation}

Physiological signals can, of course, be subject-sensitive, and have a high intra and intersubject variability as a result of age, gender, time of the day and several other factors [13]. As an illustration, different skull thickness could cause various EEG voltage levels [44]. To compensate for these types of variations, $45.5 \%$ of reviewed studies employed normalisation techniques, on either extracted features or raw signals, to enable proper comparison. However, $54.5 \%$ of the reviewed literature did not apply any normalisation techniques, in their study. So far, it has been found that researchers, in the main, have employed three different normalisation techniques (refer to Table 3 for the summary). Consider $X_{R}(t)$ as a signal recorded over time:
1. Min-Max Normalisation: The min$\max$ normalised $X_{N}(t)$ can be calculated using Equation 1 ("min" and "max" represent the minimum and maximum values of $X_{R}(t)$, respectively, over time). The extreme values of the normalised signal would be between 0 and 1. [2], [3] employed this technique to normalise the extracted features across all participants' observations, while [20], [25], [35] used this algorithm to normalise the raw recorded signals within each participant's observation (either within each window or across the entire stimuli duration).

$$
X_{N}(t)=\frac{X_{R}(t)-\min \left(X_{R}(t)\right)}{\max \left(X_{R}(t)\right)-\min \left(X_{R}(t)\right)}
$$

Equation 1 - Min-Max Normalisation Equation

2. Z-Score Normalisation: The z-score normalised $X_{N}(t)$ can be calculated using Equation 2 ("mean" and "std", represent the average and standard deviation of $X_{R}(t)$, respectively, over time). The extreme values of the normalised signal would be between about -3 and 3 [45]. [14], [24], [27], [29] employed this technique to normalise the extracted features across all participants' observations, while [15], [16], [19], [30] used this algorithm to normalise the raw recorded signals within each participant's observation (either within each window or across the entire stimuli).

$$
X_{N}(t)=\frac{X_{R}(t)-\text { mean }\left(X_{R}(t)\right)}{\operatorname{std}\left(X_{R}(t)\right)}
$$

Equation 2 - Z-Score Normalisation Equation

3. Log-Transformation: The logtransformed $X_{N}(t)$ can be calculated using Equation 3 ( $\alpha$ and $\beta$ are two constant arbitrary values). The log-transformation makes highly skewed distributions less skewed, as any $1 \%$ changes in $X_{R}(t)$ values would cause an average of $\frac{\beta}{100}$ unit changes in $X_{N}(t)$ [46]. [40] employed this technique to normalise the extracted features across all participants' observations, while [6] used this algorithm to normalise the raw recorded signals within each participant's observation (either within each window or across the entire stimuli). 


$$
X_{N}(t)=\alpha+\beta \log _{10}\left(X_{R}(t)\right)
$$

Equation 3 - Log-Transformation Equation

Implementation of normalisation techniques can be considered as an advantage for off-line classifiers, as the required features or raw signals would be extracted from the pre-recorded raw signals, before the classification process. This can provide all of the required data for the normalisation technique for the calibration process. Whereas in real-time classifiers the required features need to be extracted from the progressing raw signals, while the classification is in progress. This issue can turn the normalisation leverage to a disadvantage, as the normalisation calibration process could perform inadequately in the absence of the entire required dataset.

\subsection{Feature Selection (Dimension Reduction)}

In any affective computing system, the number of extracted psychophysiological features will increase exceedingly, as the number of physiological recordings is expanded. If the number of features were too many, the dimension of the feature vector would grow exceedingly. The feature matrix will be used in the classification and/or estimation process. Therefore the processing time would grow exponentially by increasing of the dimension of the feature vector. Moreover, high feature matrix dimensionality could cause over-fitting in the classification or estimation process, which would attenuate the accuracy considerably. To solve this problem, (I) a particular physiological signal could be discarded prior to the feature extraction process [13]. Moreover, (II) a number of features, which have either no or small contribution in explaining the affective related variations of the feature matrix, could be removed from the feature matrix [13]. The process that "removes the "irrelevant" features that do not help much with the classification problem" is known as Feature Selection or Dimension Reduction [47].

According to the reviewed literature, $48.5 \%$ of the studies have not employed any feature selection techniques, while the remainder have implemented various dimension reduction techniques, in order to perform appropriate affective recognition process. Almost half of the studies which implemented feature selection techniques, employed statistical analysis, such as Analysis of Variance (ANOVA) [3], [5], [31], [34], Chi-Square [17], Fisher's Test [10], [22], Principle Component Analysis (PCA) [21] and
Correlation Assessment [35] to reduce the dimensioanilty of the feature matrices. The other half employed other techniques, such as Sequential Forward or Backward Selection (SFS or SBS) [48] algorithm [2], [27], [30], minimalRedundancy-Maximal-Relevance (mRMR) [49] method [24], [25], Singular Value Decomposition (SVD) [50] algorithm [27], ReliefF [51] technique [24], Recursive Feature Elimination (RFE) method [18], Effect-Size (ES)-Based feature selection [52] algorithm [24] and other customised dimension reduction techniques [15], [26].

\subsection{Affective Analysis, Classification and Validation}

Only $18 \%$ of the reviewed papers investigated the significance of extracted affective features with respect to the emotional states (using t-Test, Analysis of Variance (ANOVA), correlation, etc.) [4], [28], [32], [33], [36], [40], while the remaining papers performed affective recognition. $12 \%$ of the reviewed papers employed regression algorithms to design continuous emotion detection systems [6], [14], $[\mathbf{2 3}],[\mathbf{3 5}]$. The other $70 \%$ designed and trained classifiers to detect emotional responses, according to a number of defined affective clusters. Table 4 presents the different types of classifiers employed in the reviewed studies (refer to Table 3 for the summary).

On average, studies recruited 25.5 participants $( \pm 20.54$, with a minimum of 1 and a maximum of 101), to train and validate their affective recognition system (refer to Table 3 for the summary). All studies, which were found to perform either classification or regression, employed cross-validation techniques to evaluate the performance of their affective recognition system. The studies, which implemented classification algorithms for emotion recognition processes (Table 4), reached an average of $77.91 \%$ classification accuracy $( \pm 12.76 \%$, with minimum of $50 \%$ (random classification) and maximum of $96.5 \%$ ), calculated through crossvalidation. 
Table 2 - Affective Features Related to Emotional States, Reported in the Reviewed Literature

\begin{tabular}{|c|c|c|c|}
\hline Feature & Used in Studies & Feature & Used in Studies \\
\hline $\begin{array}{l}\text { EEG Single Channel } \\
\text { Theta Rhythm }\end{array}$ & $\begin{array}{c}{[2],[3],[4],[10],[14],[18],} \\
{[23],[24],[34]}\end{array}$ & GSR Minimum & {$[2]$} \\
\hline $\begin{array}{l}\text { EEG Single Channel } \\
\text { Slow Alpha Rhythm }\end{array}$ & $\begin{array}{c}{[3],[10],[14],[18],[23]} \\
{[24]}\end{array}$ & GSR Maximum & {$[2],[19],[35],[38]$} \\
\hline $\begin{array}{l}\text { EEG Single Channel } \\
\text { Alpha Rhythm }\end{array}$ & $\begin{array}{c}{[2],[3],[4],[10],[14],[18],} \\
{[23],[24],[33],[34],[40]}\end{array}$ & GSR Standard Deviation & {$[19],[30]$} \\
\hline $\begin{array}{l}\text { EEG Single Channel } \\
\text { Beta Rhythm }\end{array}$ & $\begin{array}{c}{[2],[3],[10],[14],[18],} \\
{[23],[24],[34]}\end{array}$ & $\begin{array}{l}\text { GSR Mean of the First } \\
\text { Derivative }\end{array}$ & $\begin{array}{c}{[10],[19],[22],[23],[30],} \\
{[34],[35],[37],[38]}\end{array}$ \\
\hline $\begin{array}{l}\text { EEG Single Channel } \\
\text { Gamma Rhythm }\end{array}$ & $\begin{array}{c}{[3],[10],[14],[18],[23],} \\
{[24]}\end{array}$ & $\begin{array}{c}\text { GSR Mean of the Negative } \\
\text { Values in the First } \\
\text { Derivative }\end{array}$ & {$[10],[22],[23],[34]$} \\
\hline $\begin{array}{l}\text { EEG Paired Channel }^{3} \\
\text { Theta Rhythm }\end{array}$ & {$[3],[10],[18],[23],[24]$} & $\begin{array}{c}\text { GSR Mean of the Peak } \\
\text { Values }\end{array}$ & {$[10],[22],[23]$} \\
\hline $\begin{array}{l}\text { EEG Paired Channel }^{3} \\
\text { Slow Alpha Rhythm }\end{array}$ & {$[18],[24]$} & $\begin{array}{l}\text { GSR Low Frequency }{ }^{4} \\
\text { Spectral Power }\end{array}$ & {$[10],[22],[23]$} \\
\hline $\begin{array}{l}\text { EEG Paired Channel }^{3} \\
\text { Alpha Rhythm }\end{array}$ & {$[3],[10],[18],[23],[24]$} & Heart Rate Mean & $\begin{array}{c}{[2],[10],[17],[19],[22],} \\
{[23],[29],[30],[31],[35],} \\
{[36],[37],[38],[39]}\end{array}$ \\
\hline $\begin{array}{l}\text { EEG Paired Channel }^{3} \\
\text { Beta Rhythm }\end{array}$ & {$[3],[10],[18],[23],[24]$} & $\begin{array}{c}\text { Heart Rate Standard } \\
\text { Deviation }\end{array}$ & $\begin{array}{c}{[10],[19],[22],[23],[25],} \\
{[31],[38],[39]}\end{array}$ \\
\hline $\begin{array}{l}\text { EEG Paired Channel } \\
\text { Gamma Rhythm }\end{array}$ & {$[3],[10],[18],[23],[24]$} & $\begin{array}{c}\text { Heart Rate Mean of the } \\
\text { Peak Values }\end{array}$ & {$[35]$} \\
\hline $\begin{array}{l}\text { Asymmetric Spectral } \\
\text { Power Density Using } \\
\text { Alpha Rhythm }\end{array}$ & {$[15],[16]$} & $\begin{array}{l}\text { Heart Rate Mean of the } \\
\text { First Derivative }\end{array}$ & {$[19],[30]$} \\
\hline $\begin{array}{c}\text { Asymmetric Spectral } \\
\text { Variance Density }{ }^{6} \text { Using } \\
\text { Alpha Rhythm }\end{array}$ & {$[15],[16]$} & $\begin{array}{l}\text { Heart Rate Low Frequency }{ }^{7} \\
\text { Spectral Power }\end{array}$ & $\begin{array}{c}{[10],[17],[22],[23],[25],} \\
{[30],[31],[39]}\end{array}$ \\
\hline $\begin{array}{l}\text { Event Related Potentials } \\
\text { (EPR) }\end{array}$ & {$[5],[20],[21],[26]$} & $\begin{array}{l}\text { Heart Rate Medium } \\
\text { Frequency }{ }^{8} \text { Spectral Power }\end{array}$ & $\begin{array}{c}{[10],[17],[22],[23],[25],} \\
{[27],[30],[31],[39]}\end{array}$ \\
\hline EEGw $^{9}$ & {$[34]$} & $\begin{array}{c}\text { Heart Rate High } \\
\text { Frequency }{ }^{10} \text { Spectral Power }\end{array}$ & $\begin{array}{c}{[10],[17],[23],[25],[27],} \\
{[30],[31],[39]}\end{array}$ \\
\hline GSR Mean & $\begin{array}{c}{[2],[10],[17],[19],[22],} \\
{[23],[25],[30],[34],[35],} \\
{[36],[37],[38],[39]}\end{array}$ & $\begin{array}{l}\text { Heart Rate Spectral Power } \\
\text { Ratio }^{11}\end{array}$ & {$[10],[23],[25],[31]$} \\
\hline
\end{tabular}

\footnotetext{
Subtraction of the two channels' raw signals

${ }^{4} \mathrm{GSR}$ low frequency is $0 \mathrm{~Hz}$ to $2.4 \mathrm{~Hz}$

${ }^{5}$ Asymmetric Spectral Power Density $=\frac{P_{\text {left }}-P_{\text {right }}}{P_{\text {left }}+P_{\text {right }}}$, While "P" is the spectral power in "Alpha" frequency rhythms.

${ }^{6}$ Asymmetric Spectral Variance Density $=\frac{V_{\text {left }}-V_{\text {right }}}{V_{\text {left }}+V_{\text {right }}}$, While "V" is the spectral power in "Alpha" frequency rhythms.

${ }^{7}$ Heart Rate low frequency is $0.01 \mathrm{~Hz}$ to $0.04 \mathrm{~Hz}$.

${ }^{8}$ Heart Rate Medium frequency is $0.04 \mathrm{~Hz}$ to $0.15 \mathrm{~Hz}$.

${ }^{9} E E G_{w}=\log \left(\frac{\sum_{i=1}^{N} \beta_{i}}{\sum_{i=1}^{N}\left(\theta_{i}+\alpha_{i}\right)}\right)$, While "N" is the number of channels. $\theta, \alpha$ and $\beta$ are Theta, Alpha and Beta frequency rhythms.

${ }^{10}$ Heart Rate High frequency is $0.15 \mathrm{~Hz}$ to $0.5 \mathrm{~Hz}$.

${ }^{11}$ Heart Rate Spectral Power Ration $=\frac{\text { Medium Frequency Spectral Power }}{\text { High Frequen Spectral Power }}$
} 


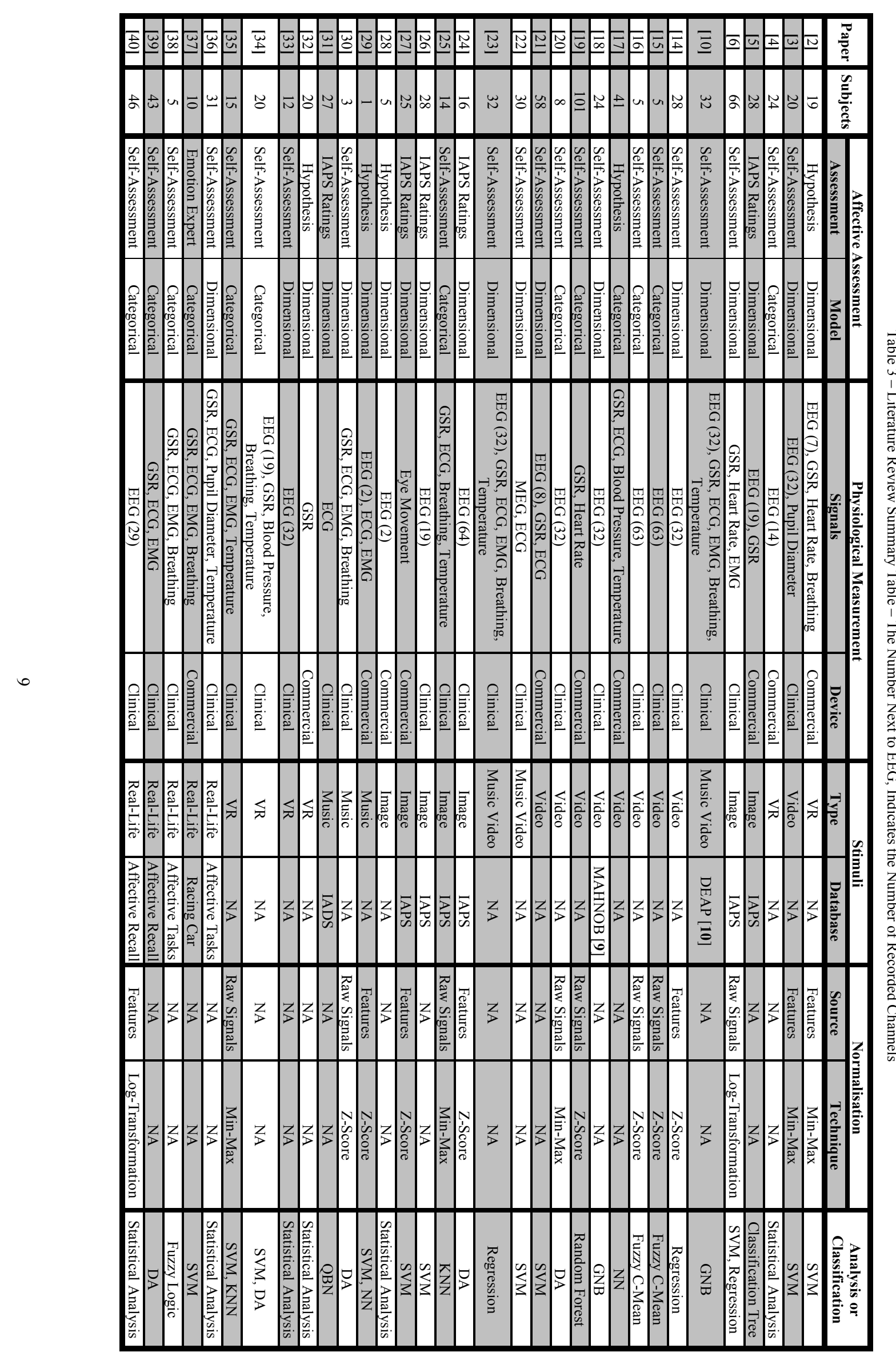


Table 4 - Classifiers Employed in Studies

\begin{tabular}{|c|c|c|c|}
\hline Classifier & Used in Studies & Classifier & Used in Studies \\
\hline $\begin{array}{c}\text { Support Vector Machine } \\
(\mathrm{SVM})\end{array}$ & $\begin{array}{c}{[\mathbf{2}],[\mathbf{3}],[\mathbf{6}],[\mathbf{2 1}],[\mathbf{2 2}],[\mathbf{2 6}],} \\
{[\mathbf{2 7}],[\mathbf{2 9}],[\mathbf{3 4}],[\mathbf{3 5}],[\mathbf{3 7}]}\end{array}$ & Neural Network $(\mathrm{NN})$ & {$[\mathbf{1 7}],[\mathbf{2 9}]$} \\
\hline $\begin{array}{c}\text { Discernment Analysis } \\
(\mathrm{DA})\end{array}$ & {$[\mathbf{2 0}],[\mathbf{2 4}],[\mathbf{3 0}],[\mathbf{3 4}],[\mathbf{3 9}]$} & $\begin{array}{c}\text { Gaussian Naïve Bayes } \\
(\mathrm{GNB})\end{array}$ & {$[\mathbf{1 0}],[\mathbf{1 8}]$} \\
\hline $\begin{array}{c}\text { Classification Tree } \\
{[\mathbf{5}],[\mathbf{3 5}],[\mathbf{3 8}]}\end{array}$ & Random Forest & {$[\mathbf{1 9}]$} \\
\hline $\begin{array}{c}\mathrm{K}^{\text {th }} \text { Nearest Neighbour } \\
(\mathrm{KNN})\end{array}$ & {$[\mathbf{2 5}],[\mathbf{3 5}]$} & $\begin{array}{c}\text { Quadratic Bayes Normal } \\
(\text { QBN) }\end{array}$ & {$[\mathbf{3 1}]$} \\
\hline Fuzzy C-Mean & {$[\mathbf{1 5}],[\mathbf{1 6}]$} & Fuzzy Logic & {$[\mathbf{3 8}]$} \\
\hline
\end{tabular}

\section{Model of Affects and Self- Assessment}

\subsection{Model of Affects}

One of the most important challenges in the study of emotion is the definition one adopts. Physiologists have presented several interpretations and definitions for emotions, and there are almost as many definitions and models for affects and emotions as there are investigators [53]. The common ground amongst all of these emotional models is that emotions impact physiological, neurophysiological and cognitive responses, to enable the individual to react and perform adequately. In high-tempo, high-pressure contexts, for example, the heart rate changes, sweating occurs, the muscles tense, facial expressions change, and many other less overt physiological changes take place facilitating the so-called "fight or flight" reaction [53]. To classify and identify the emotional experiences, two distinct affective models have been employed by the studies; Categorical (Qualitative/Discrete) and Dimensional (Quantitative/Continuous).

1. Categorical Model: The Affective Space is presented by using an emotion set (a number of 'Emotion Labels'), such that the user can be "categorised" as experiencing either one or a combination of these Emotion Labels. As an illustration, Ekman and Friesen used a categorical presentation of emotions, labelling them as surprise, fear, disgust, anger, happiness and sadness [54]. Researchers have introduced several emotion sets, although there are some common strong emotions, which are present in majority of them. These strong emotions include anger, fear, disgust, excitement, happiness, sadness and boredom [53]. Almost 40\% of the affect analysis and recognition papers reviewed in this study, employed a categorical model of emotion to perform emotional assessments $[4],[15],[16],[17],[19],[20],[25],[34]$, [35], [37], [38], [39], [40] (refer to Table 3 for the summary).

2. Dimensional Models: A number of parameters are employed to numerically present emotional experiences within a dimensional space. Both Russell and Mehrabian presented two similar dimensional models in the 1980s and 1970s. These models define emotions based on two or three continuous independent parameters (dimensions or axes) [55], [56]. Mehrabian introduced three independent quantities: Valence, defining pleasure and displeasure; Arousal describing the excitation level; and Dominance identifying the level of control within a given situation. Russell, on the other hand, ignored Dominance, and created a 2dimensional Circumplex of Affect. Mehrabian and Russell believed that representations of verbal labels of emotions, within either the 2D or 3D model, would differ between people with different cultures, especially those with different languages [55], [56]. In 1980 Russell represented some of the most common English verbal labels within his Circumplex of Affect. Figure 2 presents a simplified version of the Russell's Circumplex of Affect, occupying only eight Emotion Labels, which were distributed evenly within the model. Almost $60 \%$ of the affect analysis and recognition papers, reviewed in this study, employed the dimensional model of affects to perform emotion assessments [2], [3], [5], [6], [10], $[14],[18],[21],[22],[23],[24],[26],[27]$, [28], [29], [30], [31], [32], [33], [36] (refer to Table 3 for the summary). 


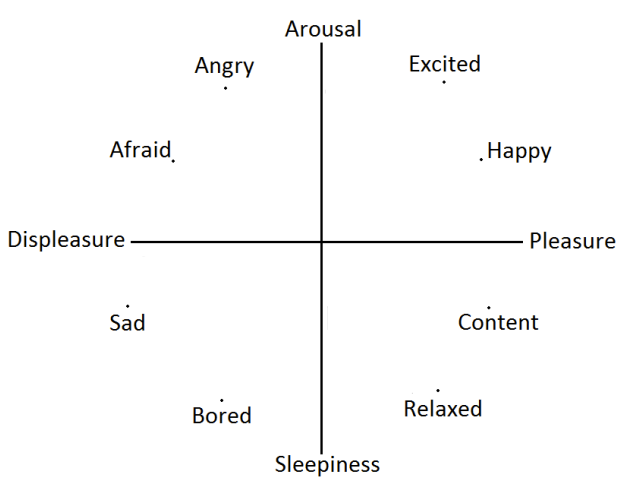

Figure 2 - Simplified Russell Circumplex of Affect for 8 English Verbal Labels of Emotions [55]

\subsection{Emotional Experience Assessment}

In the affective psychophysiological database construction process, each session, in which participants are exposed to an affective stimulus, has to be tagged by an emotional experience state, within an Affective Space (categorical and/or dimensional). This assessment has to be capable of reliably categorising the participants' emotional experience. So far studies have, in the main, employed pre-affective hypothesis, expert or self-assessments (refer to Table 3 for the summary):

1. Pre-affective hypothesis: A hypothesis is presented in the study to tag the participants' experience with an affective state [2], [17], [28], [29], [32]. As an illustration, [17] employed an emotion recognition system as an affective tagging reference, which used the participants' facial gestures to classify the emotional experience. They hypothesised that the system classification based on facial gestures is reliable enough to be used as the reference point for the physiological affective recognition system's training process. As another example, [28] hypothesised that the designed affective stimuli would evoke the target emotional experience on all participants.

2. Expert assessments: Here, a psychologist or human emotion expert is instructed to evaluate the participant's affective state, and to categorise it within an Affective Space [37].

3. Self-assessment: There are two different techniques within this category. Firstly the minority of studies employed preevaluated affective datasets ${ }^{12}$, and used the

${ }^{12}$ Such as the International Affective Picture System (IAPS) [7], or for Digital Sound (IADS) [8] stimuli's average reported affective assessments as the reference point, instead of requesting the participants to evaluate their emotional experience after each stimulus [5], [24], [26], [27], [31]. In the second technique, covering the majority of studies, participants were instructed to evaluate their emotional experience after each stimulus, rather than relying on pre-evaluated ${ }^{12}$ assessments (if available) [3], [4], [6], [10], [14], [15], [16], $[18],[19],[20],[21],[22],[23],[25],[30]$, $[33],[34],[35],[36],[38],[39],[40]$.

\subsection{Emotion Assessment in Affective Experiments}

Employing a pre-affective hypothesis can cause unreliability within the database. For instance, a stimulus, hypothesised to evoke a particular emotion, could induce a completely different emotion on the part of the users. This mismatch between the hypothesised and evoked emotion could invalidate the classification process, as there is no emotional feedback to evaluate the reliability and effectiveness of the affective stimuli. Moreover, a psychologist or human emotion expert may be unavailable to perform the emotional assessments on the participants who are exposed to affective stimuli. Therefore, employing self-assessment techniques, specifically for the purposes of emotion assessment, can be considered as the most reliable technique in the conduct of affective experiments. Indeed, and according to the findings of the literature review, the selfassessment technique has been employed by the majority of research studies $(82 \%$ of the reviewed studies).

\section{Conclusion and Future Works}

This paper serves to demonstrate that, in the domain of human-computer interaction (HCI), affect computing is ripe for exploitation in endeavours to measure users' emotions and affective experiences. In the past decades, researchers have started to contribute to this advanced and complex frontier, aiming to transform the human-computer interaction process into a completely new level of sophistication. As part of an affective computing study, this paper has conducted a deep and detailed literature review of over 33 affective computing studies undertaken since 1993, on various static, dynamic and interactive affective stimuli. It is believed that this contribution not only summarises the breadth of research over the 
past 15 years, but also serves to clarify various significant aspects and details of this increasingly valuable and relevant research area, especially as international developments in VR, gaming and related creative media pursuits gather pace and more attention is being paid to the future role of psychophysiological engagement and end user monitoring.

Moreover by presenting two types of emotion models (or so-called Affective Spaces Categorical vs. Dimensional), as have been mainly employed in this area of research, it was discovered that neither of these had any significantly higher popularity when compared to the other. This was due to the fact that $40 \%$ of the studies employed a categorical model, whilst the other $60 \%$ were based on dimensional space. No evidence was discovered in the literature regarding the superiority of one against the other. However, it was concluded that subjective selfassessments are the most popular (employed by $82 \%$ of the papers) and, according to the literature, the most reliable technique to assess and label emotional experiences, in order to perform appropriate psychophysiological signal classification.

As discussed throughout this paper, the affective computing systems, designed and evaluated since 1993, have been mainly assessed in off-line applications and according to crossvalidations. However, as the aspects of this research area are further investigated and its technical and ethical challenges are explored even further, the possibility of implementation of such systems within real-time applications grows higher. Such a development could have significant implications for the development of dynamic human-centred interface design techniques, supporting efficient human-system communication styles in a wide range of realworld applications.

\section{References}

[1] Gerben A. Van Kleef, "How Emotions Regulate Social Life," Current Directions in Psychological Science, vol. 18, no. 3, pp. 184-189, 2009.

[2] Dongrui $\mathrm{Wu}$ et al., "Optimal Arousal Identification and Classification for Affective Computing Using Physiological Signals: Virtual Reality Stroop Task," IEEE Transactions on Affective Computing, vol. 1, no. 2, pp. 109-118, July 2010.

[3] Mohammad Soleymani, Maja Pantic, and Thierry Pun, "Multimodal emotion recognition in response to videos," IEEE
Transactions on Affective Computing, vol. 3, no. 2, pp. 211-223, April 2012.

[4] Alejandro Rodríguez, Beatriz Rey, Miriam Clemente, Maja Wrzesien, and Mariano Alcañiz, "Expert Systems with Applications Assessing brain activations associated with emotional regulation during virtual reality mood induction procedures," Expert Systems with Applications, vol. 42, no. 3, pp. 16991709, February 2015.

[5] Christos A. Frantzidis et al., "On the Classification of Emotional Biosignals Evoked While Viewing Affective Pictures: An Integrated Data-Mining-Based Approach for Healthcare Applications," IEEE Transactions on Information Technology in Biomedicine, vol. 14, no. 2, pp. 309-318, March 2010.

[6] Peter J. Lang, Mark K. Gereenwald, Margaret M. Bradley, and Alfons O. Hamm, "Looking at pictures: affective, facial, visceral, and behavioral reactions," Psychophysiology, vol. 30, no. 3, pp. 26173, May 1993.

[7] P.J. Lang, M.M. Bradley, and B.N. Cuthbert, "International affective picture system (IAPS): Affective ratings of pictures and instruction manual," Gainesville, Technical Report A-8 2008.

[8] M.M. Bradley and P.J. Lang, "International affective digitized sounds (IADS): Stimuli, instruction manual and affective ratings," Gainesville, Tech. Rep. No. B-2 1999.

[9] Mohammad Soleymani, Jeroen Lichtenauer, and Thierry Pun, "A Multimodal Database for Affect Recognition and Implicit Tagging," IEEE Transactions on Affective Computing, vol. 3, no. 1, pp. 42 - 55, August 2011.

[10] Sander Koelstra et al., "DEAP: a Database for Emotion Analysis Using Physiological Signals," IEEE Transactions on Affective Computing, vol. 3, no. 1, pp. 18-31, January 2012.

[11] Mohammadhossein Moghimi, Robert J. Stone, Pia Rotshtein, and Neil Cooke, "Influencing Human Affective Responses to Dynamic Virtual Environments," Teleoperators and Virtual Environments, vol. 25, no. 2, pp. 81-107, Nobvember 2016.

[12] Margaret M. Bradley and Peter J. Lang, "Measuring Emotion, the Self-Assessment Manikin and the Semantic Differential," Pergamon Press, vol. 25, no. 1, pp. 49-59, 1994.

[13] Domen Novak, Matjaz Mihelj, and Marko Munih, "A survey of methods for data fusion and system adaptation using autonomic nervous system responses in physiological computing," Interacting with 
Computers 24, vol. 24, no. 3, pp. 154-172, 2012.

[14] Mohammad Soleymani, Sadjad AsghariEsfeden, Yun Fu, and Maja Pantic, "Analysis of EEG signals and facial expressions for continuous emotion detection," IEEE Transactions on Affective Computing, vol. 7, no. 1, pp. 17 - 28, May 2015.

[15] M. Rizon, M. Murugappan, R. Nagarajan, and S. Yaacob, "Asymmetric Ratio and FCM based Salient Channel Selection for Human Emotion Detection Using EEG," WSEAS Transactions on Signal Processing, vol. 4, no. 10, pp. 596-603, October 2008.

[16] M. Murugappan et al., "Time-Frequency Analysis of EEG Signals for Human Emotion Detection," in Springer-Verlag, Berlin, 2008, pp. 262-265.

[17] Jeremy N. Bailenson et al., "Real-time classification of evoked emotions using facial feature tracking and physiological responses," vol. 66, no. 5, pp. 303-317, May 2008.

[18] Sander Koelstra and Ioannis Patras, "Fusion of facial expressions and EEG for implicit affective tagging," Image and Vision Computing, vol. 31, no. 2, pp. 164-174, February 2013.

[19] Wanhui Wen et al., "Emotion Recognition Based on MultiVariant Correlation of Physiological Signals," IEEE Transactions on Affective Computing, vol. 5, no. 2, pp. 126-140, April 2013.

[20] Ashkan Yazdani, Jong-Seok Lee, and Touradj Ebrahimi, "Implicit Emotional Tagging of Multimedia Using EEG Signals and Brain Computer Interface," in The first SIGMM workshop on Social media, Beijing, 2009, pp. 81-88.

[21] Ramanathan Subramanian et al., "ASCERTAIN: Emotion and Personality Recognition using Commercial Sensors," IEEE Transactions on Affective Computing, no. 99, pp. 1-14, November 2016.

[22] Mojtaba Khomami Abadi et al., "DECAF: MEG-Based Multimodal Database for Decoding Affective Physiological Responses," IEEE Transactions on Affective Computing, vol. 6, no. 3, pp. 209 - 222, January 2015 .

[23] Mohammad Soleymani, Sander Koelstra, Ioannis Patras, and Thierry Pun, "Continuous emotion detection in response to music videos," in 2011 IEEE International Conference on Automatic Face \& Gesture Recognition and Workshops (FG 2011), Santa Barbara, 2011, pp. 803 808.

[24] Robert Jenke, Angelika Peer, and Martin
Buss, "Feature extraction and selection for emotion recognition from EEG," IEEE Transactions on Affective Computing, vol. 5, no. 3, pp. 327-339, July 2014.

[25] Davor Kukolja, Siniša Popović, Marko Horvat, Bernard Kovač, and Krešimir Ćosić, "Comparative analysis of emotion estimation methods based on physiological measurements for real-time applications," International Journal of Human Computer Studies, vol. 72, no. 10-11, pp. 717-727, November 2014.

[26] Christos A. Frantzidis et al., "Toward Emotion Aware Computing: An Integrated Approach Using Multichannel Neurophysiological Recordings and Affective Visual Stimuli," IEEE Transactions on Information Technology in Biomedicine, vol. 14, no. 3, pp. 589-597, May 2010.

[27] Hamed R. Tavakoli et al., "Predicting the valence of a scene from observers' eye movements," PloS one, vol. 10, no. 9, pp. 119, September 2015.

[28] Marini Othman, Abdul Wahab, Izzah Karim, Mariam Adawiah Dzulkifli, and Imad Fakhri Taha Alshaikli, "EEG Emotion Recognition Based on the Dimensional Models of Emotions," Procedia - Social and Behavioral Sciences, vol. 97, no. 1, pp. 3037, November 2013.

[29] Kazuhiko Takahashi and Akinori Tsukaguchi, "Remarks on Emotion Recognition from Multi-Modal BioPotential Signals," in Systems, Man and Cybernetics, 2003. IEEE International Conference, vol. 2, Yamaguchi Univ., Japan, 2003, pp. 1654-1659.

[30] Jonghwa Kim and Elisabeth Andre, "Emotion recognition based on physiological changes in music listening," IEEE Transactions on Pattern Analysis and Machine Intelligence, vol. 30, no. 12, pp. 2067-2083, December 2008.

[31] Mimma Nardelli, Gaetano Valenza, Alberto Greco, Antonio Lanata, and Enzo Pasquale Scilingo, "Recognizing emotions induced by affective sounds through heart rate variability," IEEE Transactions on Affective Computing, vol. 6, no. 4, pp. 385-394, October 2015.

[32] Avinash Parnandi, Youngpyo Son, and Ricardo Gutierrez-Osuna, "A ControlTheoretic Approach to Adaptive Physiological Games," in 2013 Humaine Association Conference on Affective Computing and Intelligent Interaction, Geneva, 2013, pp. 7-12.

[33] Boris Reuderink, Christian Mühl, and Mannes Poel, "Valence, arousal and dominance in the EEG during game play," 
International Journal of Autonomous and Adaptive Communications Systems, vol. 6, no. 1, pp. 45-62, January 2013.

[34] Guillaume Chanel, Cyril Rebetez, Mireille Bétrancourt, and Thierry Pun, "Emotion assessment from physiological signals for adaptation of game difficulty," IEEE Transactions on Systems, Man, and Cybernetics - Part A: Systems and Humans, vol. 41, no. 6, pp. 1052-1063, November 2011.

[35] Changchun Liu, Pramila Agrawal, Nilanjan Sarkar, and Shuo Chen, "Dynamic Difficulty Adjustment in Computer Games Through Real-Time Anxiety-Based Affective Feedback," International Journal of HumanComputer Interaction, vol. 25, no. 6, pp. 506-529, August 2009.

[36] Herbon Antje, Christian Peter, Lydia Markert, Elke Van Der Meer, and Jörg Voskamp, "Emotion studies in HCI-a new approach," in HCI International Conference, vol. 1, Las Vegas, 2005.

[37] Christos D. Katsis, Nikolaos Katertsidis, George Ganiatsas, and Dimitrios I. Fotiadis, "Toward Emotion Recognition in CarRacing Drivers: A Biosignal Processing Approach," IEEE Transactions on Systems, Man and Cybernetics, Part A: Systems and Humans, vol. 38 , no. 3, pp. 502-512, May 2008.

[38] Pramila Rani, Nilanjan Sarkar, and Julie Adams, "Anxiety-based affective communication for implicit human-machine interaction," Advanced Engineering Informatics, vol. 21, no. 3, pp. 323-334, July 2007.

[39] Pierre Rainville, Antoine Bechara, Nasir Naqvi, and Antonio R. Damasio, "Basic emotions are associated with distinct patterns of cardiorespiratory activity," International Journal of Psychophysiology, vol. 61, no. 1, pp. 5-18, July 2006.

[40] Steven K. Sutton and Richard J. Davidson, "Prefrontal Brain Asymmetry : Inhibition Systems," Psychological science, vol. 8, no. 3, pp. 204-210, May 2010.

[41] Saeid Sanei and Jonathon Chambers, "Filtering and Denoising," in EEG Signal Processing. West Sussex: John Wiley \& Sons, 2009, pp. 79-83.

[42] Saeid Sanei and Jonathon Chambers, "Brain Rhythms," in EEG Signal Processing. West Sussex: John Wiley \& Sons, 2009, pp. 1013.

[43] Kevin P. Murphy, "Introduction," in Machine Learning: A Probabilistic Perspective.: MIT Press, 2012, vol. 1, pp. 212.

[44] Mika Lehtinen, Kimmo Forsman, Jaakko
Malmivuo, and Hannu Eskola, "Effects of skull and scalp thickness on EEG," Medical \& Biological Engineering \& Computing, vol. 34, no. 1, pp. 263-264, June 1996.

[45] Erwin Kreyszig , Herbert Kreyszig , and Edward J. Norminton, "Mean. Standard Deviation. Variance. Empirical Rule," in Advanced Engineering Mathematics. USA: John Wiley \& Sons, 2010, pp. 1013-1015.

[46] David M. Lane et al., "Transformations," in Introduction to Statistics.: iTunes eBook, 2013, pp. 579-581.

[47] Kevin P. Murphy, "Feature Selection Using Mutual Information," in Machine Learning: A Probabilistic Perspective.: MIT Press, 2012, vol. 1, p. 89.

[48] A. Marcano-Cedeño, J. QuintanillaDomínguez, M.G. Cortina-Januchs, and D. Andina, "Feature selection using Sequential Forward Selection and classification applying Artificial Metaplasticity Neural Network," in IECON 2010 - 36th Annual Conference on IEEE Industrial Electronics Society, Glendale, 2010, pp. 2845-2850.

[49] Hanchuan Peng, Fuhui Long, and C. Ding, "Feature selection based on mutual information criteria of max-dependency, max-relevance, and min-redundancy," IEEE Transactions on Pattern Analysis and Machine Intelligence, vol. 27, no. 8, pp. 1226-1238, June 2005.

[50] G. H. Golub and C. Reinsch, "Singular value decomposition and least squares solutions," Numerische Mathematik, vol. 14, no. 5, pp. 403-420, April 1970.

[51] Marko Robnik-Šikonja and Igor Kononenko, "Theoretical and Empirical Analysis of ReliefF and RReliefF," Machine Learning, vol. 53, no. 1, pp. 23-69, October 2003.

[52] Yvan Saeys, Iñaki Inza, and Pedro Larrañaga, "A review of feature selection techniques in bioinformatics," Bioinformatics, vol. 23, no. 19, pp. 25072517, August 2007.

[53] Margaret M. Bradley and Peter J. Lang, "Emotion and Motivation," in Handbook of Psychophysiology, 2nd ed., L. G. Tassinary, and G. Berntson J.T. Cacioppo, Ed. New York: Cambridge University Press, 2006, pp. 581-607.

[54] Paul Ekman and Wallace V. Friesen, Unmasking The Face. Los Altos: ISHK, 2003, ISBN: 0-13-938175-9.

[55] James A. Russell, "A Circumplex Model of Affect," Journal of Personality and Social Psychology, vol. 39, no. 6, pp. 1161-1178, 1980.

[56] Albert Mehrabian, "A Semantic Space For Nonverbal Behaviour," Consulting and 
Clinical Psychology, vol. 35, no. 2, pp. 248257, October 1970.

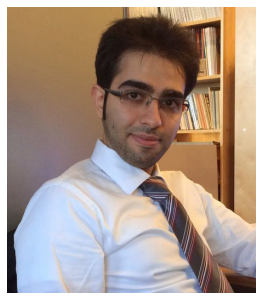

\section{Mohammadhossein} Moghimi is a teaching assistant and final-year $\mathrm{PhD}$ student in the Human Interface Technologies (HIT) research group at the School of Electronic, Electrical and System Engineering. His main research area is in implementation of BrainComputer Interaction (BCI) in Human-Computer Interaction (HCI) domain, Virtual Reality (VR) and $3 \mathrm{D}$ environments. His interests today focus on the development of Emotion Recognition system, and their implementations within virtual highly interactive environments.

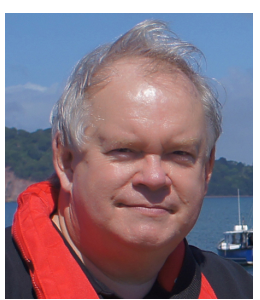

Robert (Bob) Stone is Professor of Interactive Multimedia Systems at the University of Birmingham, Director of the University's Human Interface Technologies Team, and an Academician of the Russian International Higher Education Academy of Science. A Chartered Psychologist and a Fellow of the Institute of Ergonomics and Human Factors, Bob has over 36 years of experience in human factors research and consultancy in both industry and academia. His interests today focus on the development of human-centred solutions for virtual reality, mixed reality and advanced human interface applications in defence, digital heritage and healthcare. His healthcare research focuses on future simulation and interactive technologies for post-trauma/postoperative physical and psychological rehabilitation, and future "mixed reality" predeployment training solutions for defence medics. Bob collaborates closely with the Royal Centre for Defence Medicine and the Queen Elizabeth Hospital in Birmingham, where he is also a member of the Hospital's Human Factors Faculty. The recipient of numerous national and international awards, 2017 will be Bob's $30^{\text {th }}$ year of involvement in the VR community, courtesy of a career-changing experience in 1987 at NASA Ames in California - one that has driven his human factors research goals ever since.

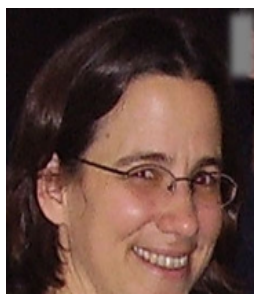

Dr. Pia Rotshtein is a cognitive neuroscientist working in the School of Psychology, University of Birmingham. Part of her areas of interest includes understanding the mechanisms underlying the superior social skills of humans, including emotional and motivational processing. 